\title{
The Radial Velocity Search for Extrasolar Planets
}

\author{
Lunar and Planetary Laboratory \\ Space Sciences Building \\ University of Arizona \\ Tucson, AZ 85721
}

Robert S. McMillan

\section{Strategy}

We are making radial velocity measurements to search for planets orbiting stars other than the Sun. The reflex acceleration induced on stars by planets can be sensed by measuring the small, slow changes in the line-of-sight velocities of stars. To detect these planetary perturbations, our data series must be made on a uniform instrumental scale for as long as it takes a planet to orbit its star. We have been operating a spectrometer of extreme stability and unprecedented sensitivity to changes in stellar radial velocities.

\section{Progress and Accomplishments}

Between December 1986 and February 1991 (inclusive) we have made 1610 observations of 16 near-solar type stars on 379 nights. (This total does not include our spectral "slope calibrations" on stars, our "check" observations of the Moon, nor about 10,000 observations of bright $\mathrm{K}$ giant stars.)

Our data show our instrument has the stability necessary to detect the reflex acceleration induced by a Jupiter-mass planet on a solar type star, if the orbit has a period less than 12 years and a favorable inclination to the line of sight. To be certain of this, we have been observing the solar spectrum reflected of a lunar crater (among other calibration sources). Our 393 observations of the Moon spread over 4 years have a standard deviation of $\pm 7 \mathrm{~m} / \mathrm{s}$, to which the random short-term "precision" contributes $\pm 5 \mathrm{~m} / \mathrm{s}$. Precision and accuracy add vectorially, so our systematic errors are $\pm 5 \mathrm{~m} / \mathrm{s}$.

Another measure of the perlormance of our instrument is the scatter of our observations of the faintest star on our program, Sigma Draconis. We have 91 observations of this K0 V star spanning 3.6 years. For detecting long-period signals it is appropriate to consider how well the averages of the four observing seasons agree. The standard deviation of the four seasonal averages is $\pm 3.3 \mathrm{~m} / \mathrm{s}$.

We have discovered new types of velocity variations intrinsic to three $\mathrm{K}$ giant stars. One binary sýstem in the literature has been refuted by our data, and another binary star system has been discovered. All these findings have been published. 


\section{Projected Accomplishments}

We will continue the observations for several nore years so that we can see at least one full planetary orbital period in the series.

We are developing a new instrument that will be more sensitive to light and more accurate than our present one.

\section{Publications}

McMillan, R. S., Smith, P. H., Perry, M. L., Moore, T. L., and Merline, W. J., 1990 Proc. SPIE 1235, Instrumentation in Astronomy VII, 601-609: "Long-term Stability of a FabryPerot Interferometer Used for Measurement of Stellar Doppler Shift". 